\title{
Introduction to Rational Functions
}

\author{
Christoph Schwarzweller \\ Institute of Computer Science \\ University of Gdańsk \\ Wita Stwosza 57, 80-952 Gdańsk, Poland
}

\begin{abstract}
Summary. In this article we formalize rational functions as pairs of polynomials and define some basic notions including the degree and evaluation of rational functions [8]. The main goal of the article is to provide properties of rational functions necessary to prove a theorem on the stability of networks.
\end{abstract}

MML identifier: RATFUNC1, version: $7.12 .02 \quad 4.181 .1147$

The notation and terminology used in this paper are introduced in the following articles: [14], [3], [4], [5], [18], [20], [16], [17], [1], [15], [2], [6], [12], [10], [11], [22], [19], [21], [9], [13], [23], and [7].

\section{Preliminaries}

One can prove the following three propositions:

(1) Let $L$ be an add-associative right zeroed right complementable right distributive non empty double loop structure, $a$ be an element of $L$, and $p, q$ be finite sequences of elements of $L$. Suppose len $p=\operatorname{len} q$ and for every element $i$ of $\mathbb{N}$ such that $i \in \operatorname{dom} p$ holds $q_{i}=a \cdot p_{i}$. Then $\sum q=a \cdot \sum p$.

(2) Let $L$ be an add-associative right zeroed right complementable right distributive non empty double loop structure, $f$ be a finite sequence of elements of $L$, and $i, j$ be elements of $\mathbb{N}$. If $i \in \operatorname{dom} f$ and $j=i-1$, then $\operatorname{Ins}\left(f_{\lceil i}, j, f_{i}\right)=f$.

(3) Let $L$ be an add-associative right zeroed right complementable associative unital right distributive commutative non empty double loop structure, $f$ be a finite sequence of elements of $L$, and $i$ be an element of $\mathbb{N}$. If $i \in \operatorname{dom} f$, then $\prod f=f_{i} \cdot \prod\left(f_{\uparrow i}\right)$.

(C) 2012 University of Białystok CC-BY-SA License ver. 3.0 or later ISSN $1426-2630(p), 1898-9934(e)$ 
Let $L$ be an add-associative right zeroed right complementable well unital associative left distributive commutative almost left invertible integral domainlike non trivial double loop structure and let $x, y$ be non zero elements of $L$. Note that $\frac{x}{y}$ is non zero.

Let us note that every add-associative right zeroed right complementable right distributive non empty double loop structure which is integral domain-like is also almost left cancelable and every add-associative right zeroed right complementable left distributive non empty double loop structure which is integral domain-like is also almost right cancelable.

Let $x, y$ be integers. Note that $\max (x, y)$ is integer and $\min (x, y)$ is integer. One can prove the following proposition

(4) For all integers $x, y, z$ holds $\max (x+y, x+z)=x+\max (y, z)$.

\section{More on Polynomials}

Let $L$ be a non empty zero structure and let $p$ be a polynomial of $L$. We say that $p$ is zero if and only if:

(Def. 1) $p=\mathbf{0} . L$.

We say that $p$ is constant if and only if:

(Def. 2) $\operatorname{deg} p \leq 0$.

Let $L$ be a non trivial zero structure. One can verify that there exists a polynomial of $L$ which is non zero.

Let $L$ be a non empty zero structure. One can verify that $\mathbf{0} . L$ is zero and constant.

Let $L$ be a non degenerated multiplicative loop with zero structure. Note that $1 . L$ is non zero.

Let $L$ be a non empty multiplicative loop with zero structure. Note that 1 . $L$ is constant.

Let $L$ be a non empty zero structure. One can verify that every polynomial of $L$ which is zero is also constant. Note that every polynomial of $L$ which is non constant is also non zero.

Let $L$ be a non trivial zero structure. One can verify that there exists a polynomial of $L$ which is non constant.

Let $L$ be a well unital non degenerated non empty double loop structure, let $z$ be an element of $L$, and let $k$ be an element of $\mathbb{N}$. Observe that $\operatorname{rpoly}(k, z)$ is non zero.

Let $L$ be an add-associative right zeroed right complementable distributive non degenerated double loop structure. One can check that Polynom-Ring $L$ is non degenerated. 
Let $L$ be an integral domain-like add-associative right zeroed right complementable distributive non trivial double loop structure. Observe that Polynom-Ring $L$ is integral domain-like.

Next we state two propositions:

(5) Let $L$ be an add-associative right zeroed right complementable right distributive associative non empty double loop structure, $p, q$ be polynomials of $L$, and $a$ be an element of $L$. Then $(a \cdot p) * q=a \cdot(p * q)$.

(6) Let $L$ be an add-associative right zeroed right complementable right distributive commutative associative non empty double loop structure, $p$, $q$ be polynomials of $L$, and $a$ be an element of $L$. Then $p *(a \cdot q)=a \cdot(p * q)$.

Let $L$ be an add-associative right zeroed right complementable well unital commutative associative distributive almost left invertible non trivial double loop structure, let $p$ be a non zero polynomial of $L$, and let $a$ be a non zero element of $L$. Note that $a \cdot p$ is non zero.

Let $L$ be an integral domain-like add-associative right zeroed right complementable distributive non trivial double loop structure and let $p_{1}, p_{2}$ be non zero polynomials of $L$. Observe that $p_{1} * p_{2}$ is non zero.

One can prove the following proposition

(7) Let $L$ be an add-associative right zeroed right complementable distributive Abelian integral domain-like non trivial double loop structure, $p_{1}, p_{2}$ be polynomials of $L$, and $p_{3}$ be a non zero polynomial of $L$. If $p_{1} * p_{3}=p_{2} * p_{3}$, then $p_{1}=p_{2}$.

Let $L$ be a non trivial zero structure and let $p$ be a non zero polynomial of $L$. One can check that degree $(p)$ is natural.

Next we state several propositions:

(8) Let $L$ be an add-associative right zeroed right complementable unital right distributive non empty double loop structure and $p$ be a polynomial of $L$. If $\operatorname{deg} p=0$, then for every element $x$ of $L$ holds eval $(p, x) \neq 0_{L}$.

(9) Let $L$ be an Abelian add-associative right zeroed right complementable well unital associative commutative distributive almost left invertible non degenerated double loop structure, $p$ be a polynomial of $L$, and $x$ be an element of $L$. Then $\operatorname{eval}(p, x)=0_{L}$ if and only if $\operatorname{rpoly}(1, x) \mid p$.

(10) Let $L$ be an Abelian add-associative right zeroed right complementable well unital associative commutative distributive almost left invertible integral domain-like non degenerated double loop structure, $p, q$ be polynomials of $L$, and $x$ be an element of $L$. If $\operatorname{rpoly}(1, x) \mid p * q$, then $\operatorname{rpoly}(1, x) \mid p$ or $\operatorname{rpoly}(1, x) \mid q$.

(11) Let $L$ be an Abelian add-associative right zeroed right complementable well unital associative commutative distributive almost left invertible non degenerated double loop structure and $f$ be a finite sequence of elements 
of Polynom-Ring $L$. Suppose that for every natural number $i$ such that $i \in \operatorname{dom} f$ there exists an element $z$ of $L$ such that $f(i)=\operatorname{rpoly}(1, z)$. Let $p$ be a polynomial of $L$. If $p=\prod f$, then $p \neq \mathbf{0}$. $L$.

(12) Let $L$ be an Abelian add-associative right zeroed right complementable well unital associative commutative distributive almost left invertible integral domain-like non degenerated double loop structure and $f$ be a finite sequence of elements of Polynom-Ring $L$. Suppose that for every natural number $i$ such that $i \in \operatorname{dom} f$ there exists an element $z$ of $L$ such that $f(i)=\operatorname{rpoly}(1, z)$. Let $p$ be a polynomial of $L$. Suppose $p=\prod f$. Let $x$ be an element of $L$. Then $\operatorname{eval}(p, x)=0_{L}$ if and only if there exists a natural number $i$ such that $i \in \operatorname{dom} f$ and $f(i)=\operatorname{rpoly}(1, x)$.

\section{Common Roots of Polynomials}

Let $L$ be a unital non empty double loop structure, let $p_{1}, p_{2}$ be polynomials of $L$, and let $x$ be an element of $L$. We say that $x$ is a common root of $p_{1}$ and $p_{2}$ if and only if:

(Def. 3) $x$ is a root of $p_{1}$ and $x$ is a root of $p_{2}$.

Let $L$ be a unital non empty double loop structure and let $p_{1}, p_{2}$ be polynomials of $L$. We say that $p_{1}$ and $p_{2}$ have a common root if and only if:

(Def. 4) There exists an element of $L$ which is a common root of $p_{1}$ and $p_{2}$.

Let $L$ be a unital non empty double loop structure and let $p_{1}, p_{2}$ be polynomials of $L$. We introduce $p_{1}$ and $p_{2}$ have common roots as a synonym of $p_{1}$ and $p_{2}$ have a common root. We introduce $p_{1}$ and $p_{2}$ have no common roots as an antonym of $p_{1}$ and $p_{2}$ have a common root.

Next we state several propositions:

(13) Let $L$ be an Abelian add-associative right zeroed right complementable unital distributive non empty double loop structure, $p$ be a polynomial of $L$, and $x$ be an element of $L$. If $x$ is a root of $p$, then $x$ is a root of $-p$.

(14) Let $L$ be an Abelian add-associative right zeroed right complementable unital left distributive non empty double loop structure, $p_{1}, p_{2}$ be polynomials of $L$, and $x$ be an element of $L$. If $x$ is a common root of $p_{1}$ and $p_{2}$, then $x$ is a root of $p_{1}+p_{2}$.

(15) Let $L$ be an Abelian add-associative right zeroed right complementable unital distributive non empty double loop structure, $p_{1}, p_{2}$ be polynomials of $L$, and $x$ be an element of $L$. If $x$ is a common root of $p_{1}$ and $p_{2}$, then $x$ is a root of $-\left(p_{1}+p_{2}\right)$.

(16) Let $L$ be an Abelian add-associative right zeroed right complementable unital distributive non empty double loop structure, $p, q$ be polynomials 
of $L$, and $x$ be an element of $L$. If $x$ is a common root of $p$ and $q$, then $x$ is a root of $p+q$.

(17) Let $L$ be an Abelian add-associative right zeroed right complementable well unital associative commutative distributive almost left invertible non trivial double loop structure and $p_{1}, p_{2}$ be polynomials of $L$. If $p_{1} \mid p_{2}$ and $p_{1}$ has roots, then $p_{1}$ and $p_{2}$ have common roots.

Let $L$ be a unital non empty double loop structure and let $p, q$ be polynomials of $L$. The common roots of $p$ and $q$ yields a subset of $L$ and is defined by:

(Def. 5) The common roots of $p$ and $q=\{x \in L: x$ is a common root of $p$ and $q\}$.

\section{Normalized Polynomials}

Let $L$ be a non empty zero structure and let $p$ be a polynomial of $L$. The leading coefficient of $p$ yields an element of $L$ and is defined by:

(Def. 6) The leading coefficient of $p=p\left(\operatorname{len} p-{ }^{\prime} 1\right)$.

We introduce LC $p$ as a synonym of the leading coefficient of $p$.

Let $L$ be a non trivial double loop structure and let $p$ be a non zero polynomial of $L$. One can check that LC $p$ is non zero.

One can prove the following proposition

(18) Let $L$ be an add-associative right zeroed right complementable well unital commutative associative distributive almost left invertible non empty double loop structure, $p$ be a polynomial of $L$, and $a$ be an element of $L$. Then $\mathrm{LC}(a \cdot p)=a \cdot \operatorname{LC} p$.

Let $L$ be a non empty double loop structure and let $p$ be a polynomial of $L$. We say that $p$ is normalized if and only if:

(Def. 7) $\quad \mathrm{LC} p=1_{L}$.

Let $L$ be an add-associative right zeroed right complementable well unital commutative associative distributive almost left invertible non trivial double loop structure and let $p$ be a non zero polynomial of $L$. One can check that $\frac{1_{L}}{\mathrm{LC} p} \cdot p$ is normalized.

Let $L$ be a field and let $p$ be a non zero polynomial of $L$. One can verify that NormPolynomial $p$ is normalized.

\section{RAtional FunCtions}

Let $L$ be a non trivial multiplicative loop with zero structure. Rational function of $L$ is defined by:

(Def. 8) There exists a polynomial $p_{1}$ of $L$ and there exists a non zero polynomial $p_{2}$ of $L$ such that it $=\left\langle p_{1}, p_{2}\right\rangle$. 
Let $L$ be a non trivial multiplicative loop with zero structure, let $p_{1}$ be a polynomial of $L$, and let $p_{2}$ be a non zero polynomial of $L$. Then $\left\langle p_{1}, p_{2}\right\rangle$ is a rational function of $L$.

Let $L$ be a non trivial multiplicative loop with zero structure and let $z$ be a rational function of $L$. Then $z_{1}$ is a polynomial of $L$. Then $z_{2}$ is a non zero polynomial of $L$.

Let $L$ be a non trivial multiplicative loop with zero structure and let $z$ be a rational function of $L$. We say that $z$ is zero if and only if:

(Def. 9) $\quad z_{1}=\mathbf{0} . L$.

Let $L$ be a non trivial multiplicative loop with zero structure. One can check that there exists a rational function of $L$ which is non zero.

Next we state the proposition

(19) Let $L$ be a non trivial multiplicative loop with zero structure and $z$ be a rational function of $L$. Then $z=\left\langle z_{1}, z_{2}\right\rangle$.

Let $L$ be an add-associative right zeroed right complementable distributive unital non trivial double loop structure and let $z$ be a rational function of $L$. We say that $z$ is irreducible if and only if:

(Def. 10) $z_{1}$ and $z_{2}$ have no common roots.

Let $L$ be an add-associative right zeroed right complementable distributive unital non trivial double loop structure and let $z$ be a rational function of $L$. We introduce $z$ is reducible as an antonym of $z$ is irreducible.

Let $L$ be an add-associative right zeroed right complementable distributive unital non trivial double loop structure and let $z$ be a rational function of $L$. We say that $z$ is normalized if and only if:

(Def. 11) $z$ is irreducible and $z_{2}$ is normalized.

Let $L$ be an add-associative right zeroed right complementable distributive unital non trivial double loop structure. Observe that every rational function of $L$ which is normalized is also irreducible.

Let $L$ be an Abelian add-associative right zeroed right complementable well unital associative distributive commutative almost left invertible integral domain-like non trivial double loop structure and let $z$ be a rational function of $L$. Note that $\operatorname{LC}\left(z_{\mathbf{2}}\right)$ is non zero.

Let $L$ be an Abelian add-associative right zeroed right complementable well unital associative distributive commutative almost left invertible integral domain-like non trivial double loop structure and let $z$ be a rational function of $L$. The norm rational function of $z$ yields a rational function of $L$ and is defined by:

(Def. 12) The norm rational function of $z=\left\langle\frac{1_{L}}{\operatorname{LC}\left(z_{\mathbf{2}}\right)} \cdot z_{\mathbf{1}}, \frac{1_{L}}{\operatorname{LC}\left(z_{\mathbf{2}}\right)} \cdot z_{\mathbf{2}}\right\rangle$.

Let $L$ be an Abelian add-associative right zeroed right complementable well unital associative distributive commutative almost left invertible integral 
domain-like non trivial double loop structure and let $z$ be a rational function of $L$. We introduce NormRatF $z$ as a synonym of the norm rational function of $z$.

Let $L$ be an Abelian add-associative right zeroed right complementable well unital associative distributive commutative almost left invertible integral domain-like non trivial double loop structure and let $z$ be a non zero rational function of $L$. Observe that the norm rational function of $z$ is non zero.

Let $L$ be a non degenerated multiplicative loop with zero structure. The functor 0 . $L$ yields a rational function of $L$ and is defined by:

(Def. 13) $0 . L=\langle\mathbf{0 .} L, \mathbf{1} . L\rangle$.

The functor 1. $L$ yields a rational function of $L$ and is defined as follows:

(Def. 14) 1. $L=\langle\mathbf{1} . L, \mathbf{1} . L\rangle$.

Let $L$ be an add-associative right zeroed right complementable distributive associative well unital non degenerated double loop structure. One can check that $0 . L$ is normalized.

Let $L$ be a non degenerated multiplicative loop with zero structure. Note that $1 . L$ is non zero.

Let $L$ be an add-associative right zeroed right complementable distributive associative well unital non degenerated double loop structure. One can verify that $1 . L$ is irreducible.

Let $L$ be an add-associative right zeroed right complementable distributive associative well unital non degenerated double loop structure. Observe that there exists a rational function of $L$ which is irreducible and non zero.

Let $L$ be an add-associative right zeroed right complementable distributive Abelian associative well unital non degenerated double loop structure and let $x$ be an element of $L$. One can check that $\langle\operatorname{rpoly}(1, x), \operatorname{rpoly}(1, x)\rangle$ is reducible and non zero as a rational function of $L$.

Let $L$ be an add-associative right zeroed right complementable distributive Abelian associative well unital non degenerated double loop structure. Observe that there exists a rational function of $L$ which is reducible and non zero.

Let $L$ be an add-associative right zeroed right complementable distributive associative well unital non degenerated double loop structure. One can verify that there exists a rational function of $L$ which is normalized.

Let $L$ be a non degenerated multiplicative loop with zero structure. One can verify that $0 . L$ is zero.

Let $L$ be an add-associative right zeroed right complementable distributive associative well unital non degenerated double loop structure. One can check that $1 . L$ is normalized.

Let $L$ be an integral domain-like add-associative right zeroed right complementable distributive non trivial double loop structure and let $p, q$ be rational functions of $L$. The functor $p+q$ yields a rational function of $L$ and is defined by: 
(Def. 15) $p+q=\left\langle p_{\mathbf{1}} * q_{\mathbf{2}}+p_{\mathbf{2}} * q_{\mathbf{1}}, p_{\mathbf{2}} * q_{\mathbf{2}}\right\rangle$.

Let $L$ be an integral domain-like add-associative right zeroed right complementable distributive non trivial double loop structure and let $p, q$ be rational functions of $L$. The functor $p * q$ yielding a rational function of $L$ is defined by:

(Def. 16) $\quad p * q=\left\langle p_{\mathbf{1}} * q_{\mathbf{1}}, p_{\mathbf{2}} * q_{\mathbf{2}}\right\rangle$.

One can prove the following proposition

(20) Let $L$ be an add-associative right zeroed right complementable well unital commutative associative distributive almost left invertible non trivial double loop structure, $p$ be a rational function of $L$, and $a$ be a non zero element of $L$. Then $\left\langle a \cdot p_{\mathbf{1}}, a \cdot p_{\mathbf{2}}\right\rangle$ is irreducible if and only if $p$ is irreducible.

\section{Normalized Rational Functions}

We now state the proposition

(21) Let $L$ be an Abelian add-associative right zeroed right complementable well unital associative distributive commutative integral domain-like non trivial double loop structure and $z$ be a rational function of $L$. Then there exists a rational function $z_{1}$ of $L$ and there exists a non zero polynomial $z_{2}$ of $L$ such that

(i) $z=\left\langle z_{2} *\left(z_{1}\right)_{\mathbf{1}}, z_{2} *\left(z_{1}\right)_{\mathbf{2}}\right\rangle$,

(ii) $z_{1}$ is irreducible, and

(iii) there exists a finite sequence $f$ of elements of Polynom-Ring $L$ such that $z_{2}=\prod f$ and for every element $i$ of $\mathbb{N}$ such that $i \in \operatorname{dom} f$ there exists an element $x$ of $L$ such that $x$ is a common root of $z_{1}$ and $z_{2}$ and $f(i)=\operatorname{rpoly}(1, x)$.

Let $L$ be an Abelian add-associative right zeroed right complementable well unital associative distributive commutative almost left invertible integral domain-like non trivial double loop structure and let $z$ be a rational function of $L$. The functor $\mathrm{NF} z$ yielding a rational function of $L$ is defined by:

(Def. 17)(i) There exists a rational function $z_{1}$ of $L$ and there exists a non zero polynomial $z_{2}$ of $L$ such that $z=\left\langle z_{2} *\left(z_{1}\right)_{1}, z_{2} *\left(z_{1}\right)_{2}\right\rangle$ and $z_{1}$ is irreducible and $\mathrm{NF} z=$ the norm rational function of $z_{1}$ and there exists a finite sequence $f$ of elements of Polynom-Ring $L$ such that $z_{2}=\prod f$ and for every element $i$ of $\mathbb{N}$ such that $i \in \operatorname{dom} f$ there exists an element $x$ of $L$ such that $x$ is a common root of $z_{\mathbf{1}}$ and $z_{\mathbf{2}}$ and $f(i)=\operatorname{rpoly}(1, x)$ if $z$ is non zero,

(ii) $\mathrm{NF} z=0 . L$, otherwise.

Let $L$ be an Abelian add-associative right zeroed right complementable well unital associative distributive commutative almost left invertible integral 
domain-like non trivial double loop structure and let $z$ be a rational function of $L$. Observe that $\mathrm{NF} z$ is normalized and irreducible.

Let $L$ be an Abelian add-associative right zeroed right complementable well unital associative distributive commutative almost left invertible integral domain-like non trivial double loop structure and let $z$ be a non zero rational function of $L$. One can verify that $\mathrm{NF} z$ is non zero.

One can prove the following propositions:

(22) Let $L$ be an Abelian add-associative right zeroed right complementable well unital associative distributive commutative almost left invertible integral domain-like non trivial double loop structure, $z$ be a non zero rational function of $L, z_{1}$ be a rational function of $L$, and $z_{2}$ be a non zero polynomial of $L$. Suppose that

(i) $z=\left\langle z_{2} *\left(z_{1}\right)_{1}, z_{2} *\left(z_{1}\right)_{2}\right\rangle$,

(ii) $z_{1}$ is irreducible, and

(iii) there exists a finite sequence $f$ of elements of Polynom-Ring $L$ such that $z_{2}=\prod f$ and for every element $i$ of $\mathbb{N}$ such that $i \in \operatorname{dom} f$ there exists an element $x$ of $L$ such that $x$ is a common root of $z_{1}$ and $z_{2}$ and $f(i)=\operatorname{rpoly}(1, x)$.

Then NF $z=$ the norm rational function of $z_{1}$.

(23) Let $L$ be an Abelian add-associative right zeroed right complementable well unital associative distributive commutative almost left invertible integral domain-like non trivial double loop structure. Then NF 0. $L=0$. $L$.

(24) Let $L$ be an Abelian add-associative right zeroed right complementable well unital associative distributive commutative almost left invertible integral domain-like non trivial double loop structure. Then NF 1. $L=1$. L.

(25) Let $L$ be an Abelian add-associative right zeroed right complementable well unital associative distributive commutative almost left invertible integral domain-like non trivial double loop structure and $z$ be an irreducible non zero rational function of $L$. Then $\mathrm{NF} z=$ the norm rational function of $z$.

(26) Let $L$ be an Abelian add-associative right zeroed right complementable well unital associative distributive commutative almost left invertible integral domain-like non trivial double loop structure, $z$ be a rational function of $L$, and $x$ be an element of $L$. Then $\operatorname{NF}\left\langle\operatorname{rpoly}(1, x) * z_{\mathbf{1}}\right.$, $\left.\operatorname{rpoly}(1, x) * z_{\mathbf{2}}\right\rangle=\mathrm{NF} z$.

(27) Let $L$ be an Abelian add-associative right zeroed right complementable well unital associative distributive commutative almost left invertible integral domain-like non trivial double loop structure and $z$ be a rational function of $L$. Then NF NF $z=\mathrm{NF} z$.

(28) Let $L$ be an Abelian add-associative right zeroed right complementable well unital associative distributive commutative almost left invertible in- 
tegral domain-like non degenerated double loop structure and $z$ be a non zero rational function of $L$. Then $z$ is irreducible if and only if there exists an element $a$ of $L$ such that $a \neq 0_{L}$ and $\left\langle a \cdot z_{\mathbf{1}}, a \cdot z_{\mathbf{2}}\right\rangle=\mathrm{NF} z$.

\section{Degree of Rational Functions}

Let $L$ be an Abelian add-associative right zeroed right complementable well unital associative distributive commutative almost left invertible integral domain-like non trivial double loop structure and let $z$ be a rational function of $L$. The functor degree $(z)$ yielding an integer is defined as follows:

(Def. 18) $\operatorname{degree}(z)=\max \left(\operatorname{degree}\left((\mathrm{NF} z)_{1}\right)\right.$, degree $\left.\left((\mathrm{NF} z)_{\mathbf{2}}\right)\right)$.

Let $L$ be an Abelian add-associative right zeroed right complementable well unital associative distributive commutative almost left invertible integral domain-like non trivial double loop structure and let $z$ be a rational function of $L$. We introduce $\operatorname{deg} z$ as a synonym of $\operatorname{degree}(z)$.

Next we state two propositions:

(29) Let $L$ be an Abelian add-associative right zeroed right complementable well unital associative distributive commutative almost left invertible integral domain-like non trivial double loop structure and $z$ be a rational function of $L$. Then degree $(z) \leq \max \left(\operatorname{degree}\left(z_{1}\right)\right.$, degree $\left.\left(z_{2}\right)\right)$.

(30) Let $L$ be an Abelian add-associative right zeroed right complementable well unital associative distributive commutative almost left invertible integral domain-like non trivial double loop structure and $z$ be a non zero rational function of $L$. Then $z$ is irreducible if and only if $\operatorname{degree}(z)=\max \left(\operatorname{degree}\left(z_{1}\right), \operatorname{degree}\left(z_{2}\right)\right)$.

\section{Evaluation of Rational Functions}

Let $L$ be a field, let $z$ be a rational function of $L$, and let $x$ be an element of $L$. The functor eval $(z, x)$ yielding an element of $L$ is defined by:

(Def. 19) $\operatorname{eval}(z, x)=\frac{\operatorname{eval}\left(z_{1}, x\right)}{\operatorname{eval}\left(z_{2}, x\right)}$.

The following propositions are true:

(31) For every field $L$ and for every element $x$ of $L$ holds eval(0. $L, x)=0_{L}$.

(32) For every field $L$ and for every element $x$ of $L$ holds eval(1. $L, x)=1_{L}$.

(33) Let $L$ be a field, $p, q$ be rational functions of $L$, and $x$ be an element of $L$. If $\operatorname{eval}\left(p_{\mathbf{2}}, x\right) \neq 0_{L}$ and $\operatorname{eval}\left(q_{\mathbf{2}}, x\right) \neq 0_{L}$, then $\operatorname{eval}(p+q, x)=\operatorname{eval}(p, x)+$ $\operatorname{eval}(q, x)$.

(34) Let $L$ be a field, $p, q$ be rational functions of $L$, and $x$ be an element of $L$. If eval $\left(p_{\mathbf{2}}, x\right) \neq 0_{L}$ and $\operatorname{eval}\left(q_{\mathbf{2}}, x\right) \neq 0_{L}$, then $\operatorname{eval}(p * q, x)=\operatorname{eval}(p, x)$. $\operatorname{eval}(q, x)$. 
(35) Let $L$ be a field, $p$ be a rational function of $L$, and $x$ be an element of $L$. If $\operatorname{eval}\left(p_{\mathbf{2}}, x\right) \neq 0_{L}$, then eval(the norm rational function of $\left.p, x\right)=\operatorname{eval}(p, x)$.

(36) Let $L$ be a field, $p$ be a rational function of $L$, and $x$ be an element of $L$. If $\operatorname{eval}\left(p_{\mathbf{2}}, x\right) \neq 0_{L}$, then $x$ is a common root of $p_{\mathbf{1}}$ and $p_{\mathbf{2}}$ or eval(NF $\left.p, x\right)=$ $\operatorname{eval}(p, x)$.

\section{REFERENCES}

[1] Grzegorz Bancerek. The fundamental properties of natural numbers. Formalized Mathematics, 1(1):41-46, 1990.

[2] Grzegorz Bancerek. The ordinal numbers. Formalized Mathematics, 1(1):91-96, 1990.

[3] Grzegorz Bancerek and Krzysztof Hryniewiecki. Segments of natural numbers and finite sequences. Formalized Mathematics, 1(1):107-114, 1990.

[4] Czesław Byliński. Functions and their basic properties. Formalized Mathematics, 1(1):5565, 1990.

[5] Czesław Byliński. Functions from a set to a set. Formalized Mathematics, 1(1):153-164, 1990.

[6] Czesław Byliński. Partial functions. Formalized Mathematics, 1(2):357-367, 1990.

[7] Czesław Byliński. Some basic properties of sets. Formalized Mathematics, 1(1):47-53, 1990.

[8] H. Heuser. Lehrbuch der Analysis. B.G. Teubner Stuttgart, 1990.

[9] Eugeniusz Kusak, Wojciech Leończuk, and Michał Muzalewski. Abelian groups, fields and vector spaces. Formalized Mathematics, 1(2):335-342, 1990.

[10] Robert Milewski. The evaluation of polynomials. Formalized Mathematics, 9(2):391-395, 2001.

[11] Robert Milewski. Fundamental theorem of algebra. Formalized Mathematics, 9(3):461470, 2001.

[12] Robert Milewski. The ring of polynomials. Formalized Mathematics, 9(2):339-346, 2001.

[13] Michał Muzalewski. Construction of rings and left-, right-, and bi-modules over a ring. Formalized Mathematics, 2(1):3-11, 1991.

[14] Michał Muzalewski and Lesław W. Szczerba. Construction of finite sequences over ring and left-, right-, and bi-modules over a ring. Formalized Mathematics, 2(1):97-104, 1991.

[15] Jan Popiołek. Real normed space. Formalized Mathematics, 2(1):111-115, 1991.

[16] Christoph Schwarzweller and Agnieszka Rowińska-Schwarzweller. Schur's theorem on the stability of networks. Formalized Mathematics, 14(4):135-142, 2006, doi:10.2478/v10037006-0017-9.

[17] Michał J. Trybulec. Integers. Formalized Mathematics, 1(3):501-505, 1990.

[18] Wojciech A. Trybulec. Groups. Formalized Mathematics, 1(5):821-827, 1990.

[19] Wojciech A. Trybulec. Vectors in real linear space. Formalized Mathematics, 1(2):291-296, 1990.

[20] Wojciech A. Trybulec. Lattice of subgroups of a group. Frattini subgroup. Formalized Mathematics, 2(1):41-47, 1991.

[21] Zinaida Trybulec. Properties of subsets. Formalized Mathematics, 1(1):67-71, 1990.

[22] Edmund Woronowicz. Relations and their basic properties. Formalized Mathematics, 1(1):73-83, 1990.

[23] Hiroshi Yamazaki and Yasunari Shidama. Algebra of vector functions. Formalized Mathematics, 3(2):171-175, 1992.

Received February 8, 2012 